\title{
Intrinsic Multichannel Power Control in Cascaded Power-Equalizing Erbium-Doped Fiber Amplifiers
}

\author{
J. Nilsson
}

\begin{abstract}
We numerically investigate the performance of a cascade of four-channel power-equalizing reflective erbium-doped fiber amplifiers (EDFAs). The EDFAs reflect different channels at different points so that different channels interact with partly different sections of the EDFA. Thus, the gain for different channels saturate partly independently. The channels can self-heal independently in the cascade, which therefore is less sensitive to harmful channel-dependent and channel-independent inter-amplifier loss variations than a cascade of normal, nonequalizing, EDFAs is. Moreover, the design channel-power is maintained even if channels are dropped. In contrast to a cascade of typical nonequalizing EDFAs, we can in addition control the channel power evolution via the pumping of the power-equalizing EDFAs.
\end{abstract}

Index Terms-Erbium, optical communications, optical equalizers, optical fiber amplifiers, wavelength division multiplexing.

W AVELENGTH-DIVISION-MULTIPLEXED (WDM) transmission through a cascade of optical amplifiers is vulnerable to detrimental spreading of the channel-powers [1]-[5]. This motivates the use of gain-flattening filters [4]. However, except with complex and expensive adjustable filters [5], the filters will not bring the channel-powers together if such a power spread anyhow arises, e.g., as a consequence of wavelength routing or of wavelength-dependent variations of the inter-amplifier loss. On the other hand, power-equalizing optical amplifiers [1]-[3], [6] can stop the power from spreading in the first place, and eliminate a pre-existing spread. Since the gain-spectrum of an erbium-doped fiber (EDF) is predominantly homogeneously broadened at room temperature, power-equalization is difficult in an erbium-doped fiber amplifier (EDFA). However, it is still possible if different wavelengths travel different paths through the amplifier [2], [3], [6]. We have previously proposed and theoretically investigated a four-channel power-equalizing EDFA in which different channels are reflected at different points [6]. In this letter, we numerically simulate cascades of such EDFAs when the span loss deviates from its nominal value.

Fig. 1 depicts the EDFA. Because different channels interact with different parts of the EDFA to some extent, the gain can saturate partly independently for different channels, even though the different EDF segments are assumed to be homogeneously broadened. The EDFA is described more closely in [6]. The fiber gratings that reflect the different channels each have a bandwidth of $0.8 \mathrm{~nm}(100 \mathrm{GHz})$. The gratings are perfectly re-

Manuscript received August 9, 1999; revised April 28, 2000.

The author was with Samsung Electronics, Optical Communications R\&D Group, Information and Communications Business, Suwon, Korea 440-600. He is now with the Optoelectronics Research Centre, University of Southampton, Southampton SO17 1BJ, U.K. (e-mail: jn@orc.soton.ac.uk).

Publisher Item Identifier S 1041-1135(00)06288-1.

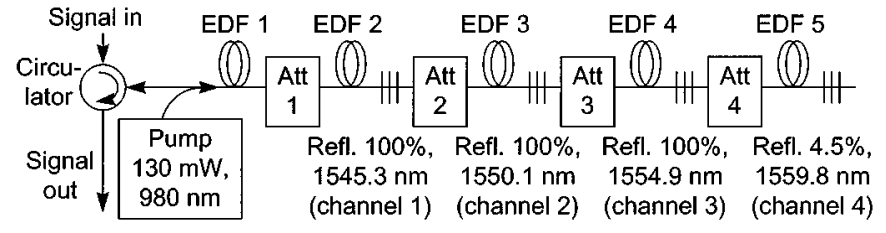

Fig. 1. Schematic drawing of reflective power-equalizing EDFA. The attenuators are fixed but wavelength-dependent, and transparent to the pump.

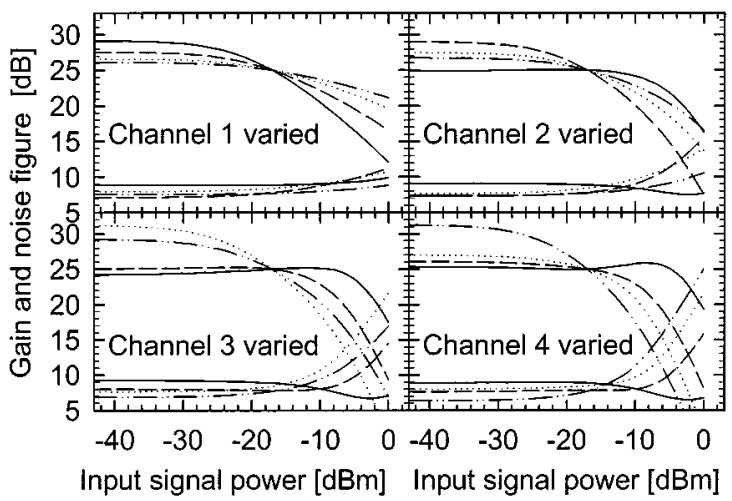

Fig. 2. Gain and noise figure of a single EDFA as the input power deviates from its nominal $-17 \mathrm{dBm}$ for each of the four channels in succession. The input powers of the other three channels were in each case kept at $-17 \mathrm{dBm}$. Line legend: channel 1 solid, channel 2 dashed, channel 3 dotted, channel 4 chain-dashed.

flecting except the last one at $1559.8 \mathrm{~nm}$, which has a $13.5 \mathrm{~dB}$ reflection loss $(R=4.5 \%)$. The gain of the last segment (amplifying only the $1559.8 \mathrm{~nm}$ signal) is correspondingly higher. The higher gain increases the latitude for gain compression, which promotes equalization. At the same time, a too low reflectivity would make the reflected signal power so low that the signal-to-noise ratio would degrade.The EDFA gain is $25 \mathrm{~dB}$ for an input power of $-17 \mathrm{dBm}$ in each channel. This matches the nominal span loss in the cascades we consider. The relatively low inversion at the input of reflective EDFAs and the strong attenuation make the noise figures high, 7.7-8.9 $\mathrm{dB}$ for the different channels at $-17 \mathrm{dBm}$ channel input power. A pre-amplifier stage before the circulator would reduce the noise figure. Fig. 2 illustrates how the EDFA gain and noise figure varies if the input power deviates in any of the four channels. In all cases, a 1-dB input power spread is equalized (reduced) by between $20 \%$ and $80 \%$ without requiring any adjustable filters, pump power control, or monitoring of powers [6].

Turning next to EDFA cascades, Fig. 3 shows how signal and ASE powers evolve when channel 2 suffers an excess loss of $15 \mathrm{~dB}$ in the span following the first amplifier. Nevertheless, its power recovers to that of the other channels after $\sim 10$ stages 
of power-equalizing EDFAs [Fig. 3(a)]. The higher gain for channel 2 also leads to more ASE there, since the ASE from an EDFA is proportional to the gain (and the noise figure, which, however, does not change much with a input power reduction). ASE accumulation causes the slow drop in signal power along the cascade. A smaller reflection bandwidth would reduce the ASE accumulation. The larger ASE-power at channel 2 explains the slight remaining signal power difference of $0.6 \mathrm{~dB}$ between the perturbed and unperturbed channels - the power equalization does not distinguish ASE from signal power. After 50 spans, the optical signal-to-noise ratios (SNR) vary between 13.4 (for the perturbed channel) and $16.8 \mathrm{~dB}$ (throughout this paper, we use a noise bandwidth of $10 \mathrm{GHz}$ for the optical SNR). The ASE power differences are larger than the signal power differences and are the main source of the SNR differences. For an unperturbed cascade, the SNR is $15.6-16.8 \mathrm{~dB}$. The $1.2 \mathrm{~dB}$ channel variation equals the difference in noise figure for a single amplifier. In contrast to channel-dependent gain variations, noise figure variations do not build up in a cascade and are therefore less of a problem. The cascade self-healed similarly also if another channel than \#2 was perturbed. For comparison, Fig. 3(b) shows what happens in a corresponding cascade of nonequalizing EDFAs for the same perturbation. These EDFAs resemble the power-equalizing EDFA of Fig. 1, but they have only a single EDF-segment followed by four Bragg gratings with reflectivities chosen so that the EDFA gain becomes $25 \mathrm{~dB}$ for all channels at $-17 \mathrm{dBm}$ of channel input power. Because the EDF-segment is common to all channels, the gain is homogeneously broadened. All channels experience approximately the same gain even after the perturbation, and the power of the channel with the excess loss does not recover in Fig. 3(b). The similar gains lead to similar ASE-levels. The large spread of signal powers dominate the SNR spread.

Fig. 4 is similar to Fig. 3, but now the channel 2 excess loss is $1 \mathrm{~dB}$ in each span. With power-equalizing EDFAs [Fig. 4(a)], the channel power spread is smaller than $4 \mathrm{~dB}$ throughout the cascade despite the $50 \mathrm{~dB}$ difference in total loss between channels. It follows that the EDFAs equalized an input power difference by more than $1 \mathrm{~dB} / 4 \mathrm{~dB}=25 \%$. The SNR spread is smaller than $3 \mathrm{~dB}$. In stark contrast to this, the power spread is $60.1 \mathrm{~dB}$ after the cascade of nonequalizing EDFAs [Fig. 4(b)]. To conclude our treatment of single-channel perturbations, Fig. 5 illustrates how the optical SNR of the perturbed channel (which is noisiest) depends on the size of the excess loss for different perturbed channels and types of EDFAs. In Fig. 5(a), a single span is perturbed, while all spans are perturbed in Fig. 5(b).

Next, we consider cascades in which the span-loss deviates from the nominal $25 \mathrm{~dB}$ by the same amount in each of the four channels. The signal powers then evolve to new levels, while the operating gain of the EDFA adjusts to the perturbed span loss. Unfortunately, the channel gains of EDFAs are typically only equalized for a specific value of the operating gain, so when this changes, the channel gains will differ. With nonequalizing EDFAs, the gain differences will accumulate to a large spread of channel-powers and a degraded SNR, as illustrated in Fig. 6(b). On the other-hand, the equalizing EDFAs used for Fig. 6(a) keep the power-spread in check, and the cascade works over a much larger range of span losses. Still, the SNR (and also power) of

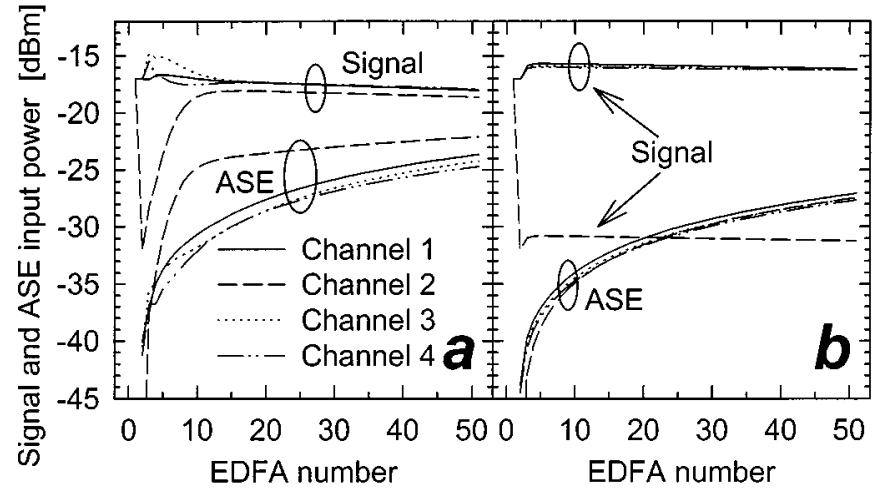

Fig. 3. Evolution of signal and ASE power at the input of the EDFAs in a cascade of 50 amplifiers. Channel 2 has an excess loss of $15 \mathrm{~dB}$ between the first and second EDFA. (a) Cascade of power-equalizing EDFAs. (b) Cascade of nonequalizing EDFAs.

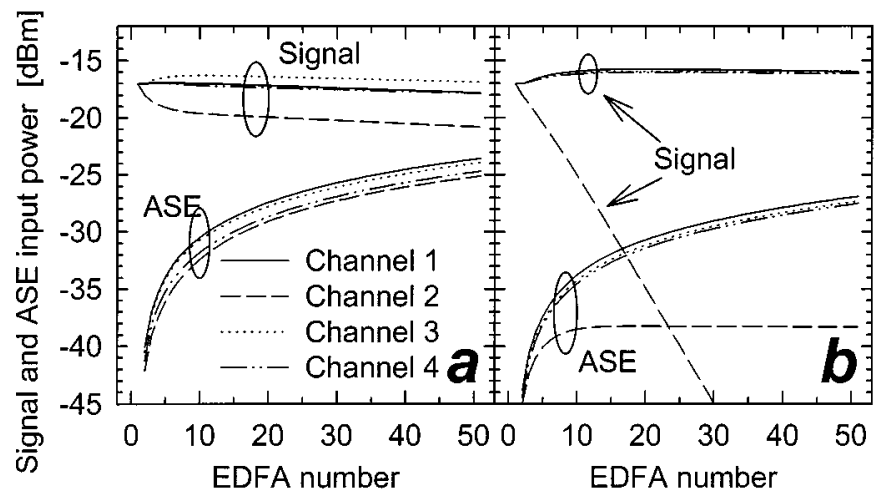

Fig. 4. Evolution of signal and ASE power at the input of the EDFAs in a cascade of 50 amplifiers. Channel 2 has an excess loss of $1 \mathrm{~dB}$ in each span. (a) Cascade of power-equalizing EDFAs. (b) Cascade of nonequalizing EDFAs.

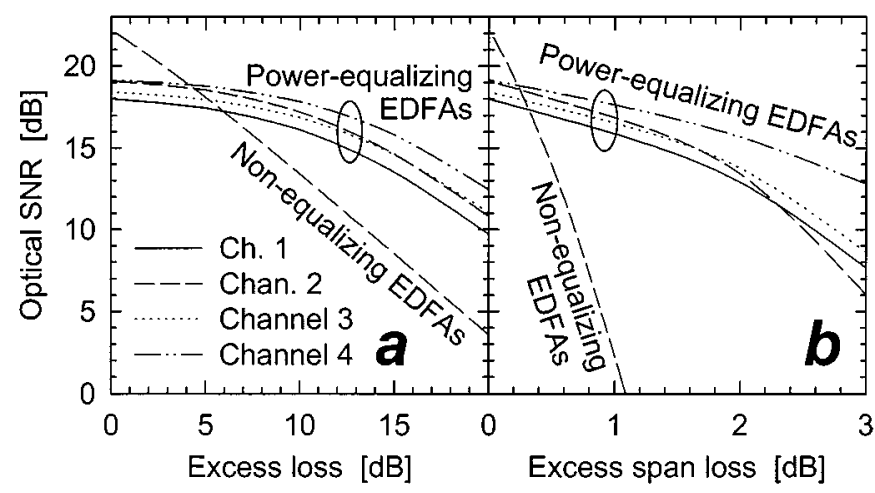

Fig. 5. Optical SNR of perturbed channel vs. amount of perturbation after 30-EDFA cascades of equalizing and nonequalizing EDFAs. For cascades of power-equalizing EDFAs, the figure shows all four channels perturbed in succession, while for cascades nonequalizing EDFAs, only a perturbation of channel 2 is shown; the other channels behaved similarly when perturbed. (a) Perturbation is an excess loss of a single channel in the span following the first EDFA only (as in Fig. 3). (b) Perturbation is an excess loss of a single channel in each of the spans (as in Fig. 4).

channel 1 does vary, and drops rapidly for span losses above 28 dB [Fig. 6(a)]. Because channel 1 does not penetrate that far into the EDFA, it is less affected by gain saturation than the other channels are. Thus, the gain of channel 1 responds relatively weakly when all channel powers are varied together. For a 1-dB change of channel input power from $-17 \mathrm{dBm}$, the gain changes 
by $0.48,1.12,1.51$, and $1.64 \mathrm{~dB}$ for channels $1-4$, respectively. Hence, compared to channel 1, the gains of channels 2-4 will more readily increase to compensate a larger span loss (with resulting lower input powers).

There are several options for reducing the excessive sensitivity of channel 1 . We can use two different designs of powerequalizing EDFAs alternatingly in the cascade. The EDFAs are similar, except that the reflection orders of the channels are opposite in the different types of EDFAs. Thus, on average, the channels penetrate equally far into the EDFAs. Two results for such a cascade are also shown in Fig. 6(a) for the noisiest channel (channel 1). Two alternative methods require, in contrast to the other results in this paper, some parameters to be adjusted according to the operating conditions of the EDFA. The methods rely on the possibility to manipulate the gain spectrum of the power-equalizing EDFA by pumping different EDFsegments differently. One method requires two pump sources, launched from opposite ends of the EDFA. Then, the gain of the channel penetrating the farthest into the EDFA can be reduced by reducing the pump power launched in the tail end of the EDFA. For our single-pumped EDFA, one can instead tune the pump wavelength. In case of a grating-stabilized $980 \mathrm{~nm}$ pump diode, stretching the fiber grating will tune the diode. If we tune the pump to wavelengths with lower or higher absorption, we can control the power that reaches the farthest-lying segments. Fig. 6(a) includes two results for the noisiest channel (\#1) that show possible improvements. We note that if the wavelength-dependence of the pump-absorption differs from EDF to EDF, the diodes to some extent could be addressed to pump different EDF-segments. In an extreme case, each EDF-segment could be separately pumped. The channel gains cannot be manipulated like this if all channels travel the same paths through homogeneously broadened EDF's of the same type [7].

Let us briefly consider what happens if channels are dropped. We only treat the steady-state power evolution and ignore any transients [7]. In a nonequalizing EDFA, the power of a dropped channel is redistributed among the remaining ones. If three of four channels are dropped from a system, the power in the surviving channel increases by $6 \mathrm{~dB}$, which can cause problems with fiber nonlinearities and may exceed the dynamic range of the receiver. We can counter this by reducing the pump power as channels are dropped, but the number of dropped channels may be unknown and difficult to measure. With the powerequalizing EDFAs, the power of remaining channels automatically assumes the design value $\sim 10$ spans downstream the perturbation without any pump power adjustment. At the same time, ASE builds up at the missing channel(s) to compensate the missing channel-power.

The power-equalizing EDFAs can compensate various perturbations in a network and are in that sense more flexible than nonequalizing ones. Nevertheless, any compensation is limited in its scope and degrades performance. Like standard EDFAs, power-equalizing EDFAs should therefore be designed to the nominal operating conditions. Different systems may require gain below $10 \mathrm{~dB}$ or above $30 \mathrm{~dB}$ with different channel powers. We have not investigated to what extent we can adapt the powerequalizing EDFAs to different nominal operating conditions, but we believe that they can reach at least $30 \mathrm{~dB}$ of gain (if necessary

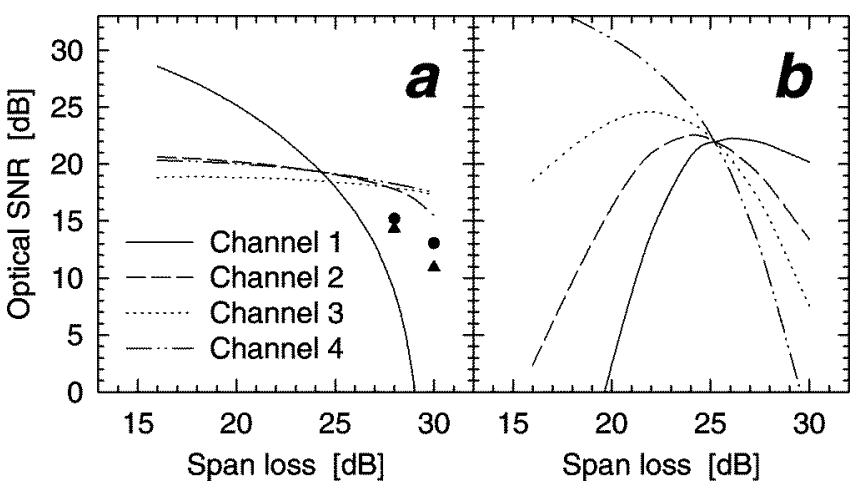

Fig. 6. Optical SNR versus span loss for 30-amplifier cascades of (a) equalizing EDFAs (left) and (b) nonequalizing EDFAs. Results are also shown for the noisiest channel (\#1) for a cascade of alternatingly different equalizing EDFAs (triangles) and for a cascade of equalizing EDFAs in which the pump wavelength was adjusted for highest SNR (circles).

we could use a pre-amplifier). Lower gains should be possible too, as long as the signal powers become large enough to saturate the EDFAs. Power equalization depends on saturation and will therefore not work for too low powers. A large number of channels makes a power-equalizing EDFA very complex. Moreover, the influence that a single channel has on the EDFA gain becomes smaller with more channels, making the power equalization less effective with more channels. Our power-equalizing EDFAs are also obviously restricted to operate at specific wavelengths. It would not be practicable to add new wavelengths to installed EDFAs.

In summary, we numerically investigated the performance of a cascade of four-channel reflective intrinsically power-equalizing EDFAs. Such a cascade is less sensitive to channel-dependent and channel-independent changes of the inter-amplifier loss than a cascade of nonequalizing EDFAs is. It can also maintain the design channel-power even if channels are dropped. The power-equalizing EDFAs are more complex than standard EDFAs, but are also more amenable to active control of the gain spectra.

\section{REFERENCES}

[1] E. L. Goldstein, L. Eskildsen, V. da Silva, M. Andrejco, and Y. Silberberg, "Inhomogeneously broadened fiber-amplifier cascades for transparent multiwavelength networks," J. Lightwave Technol., vol. 13, pp. 782-790, 1995.

[2] M. N. Zervas and R. I. Laming, "Twin-core fiber erbium-doped channel equalizers," J. Lightwave Technol., vol. 13, pp. 721-731, 1995.

[3] L. Eskildsen, E. L. Goldstein, G. K. Chang, M. Z. Iqbal, and C. Lin, "Self-regulating WDM amplifier module for scalable lightwave networks," IEEE Photon. Technol. Lett., vol. 6, pp. 1321-1323, 1994.

[4] A. M. Vengsarkar, J. R. Pedrazzini, J. B. Judkins, P. J. Lemaire, N. S. Bergano, and C. R. Davidson, "Long-period fiber-grating-based gain equalizers," Opt. Lett., vol. 21, pp. 336-338, 1996.

[5] S. M. Hwang and A. E. Willner, "Active equalization of nonuniform EDFA gain by using multiple AOTF passbands for megameter WDM transmission," in Conference on Lasers and Electro-Optics, ser. OSA Technical Digest Series. Washington, D.C.: Opt. Soc. Amer., 1995, vol. 15 , pp. 160-161.

[6] J. Nilsson, J. M. Kim, Y. W. Lee, S. T. Hwang, S. Y. Yoon, and S. J. Kim, "Multi-channel power-equalizing reflective erbium-doped fiber amplifier," in Pacific Rim Conference on Lasers and Electro-Optics. New York: IEEE Press, 1999, vol. 3, pp. 652-653.

[7] Y. Sun, J. L. Zyskind, and A. K. Srivastava, "Average inversion level, modeling, and physics of erbium-doped fiber amplifiers," IEEE J. Select. Topics Quantum Electron., vol. 3, pp. 991-1007, 1997. 